\title{
2. ELECCIONES EN AMÉRICA LATINA
}

\section{ELECCIONES, ELECTORES Y PARTIDOS EN AMERICA LATINA EN LA DECADA DE 1990}

\section{Manuel Alcántara Saez*}

\section{INTRODUCCION}

Una de las características más sobresalientes que comportan los procesos de desarrollo democrático que se ha generalizado en la última década en los países latinoamericanos, salvo en Cuba, ha sido la extensión de la práctica electoral. La proliferación de elecciones libres y competitivas, funcionales para los sistemas políticos democráticos, en el sentido de la operatividad de los mismos al canalizar la participación y representación de los ciudadanos, y asegurar reemplazos ordenados de la clase política en el poder, es una constante de los tiempos que corren que les diferencian significativamente de etapas pretéritas. Si bien la bondad de las elecciones es acogida en grados muy diferentes por parte de los ciudadanos de distintos países latinoamericanos, el papel que desempeñan los comicios "para que las cosas sean diferentes en el futuro" o el conferido a los partidos para que con ellos "pueda haber democracia", es mayoritario (1).

Desde una perspectiva neoinstitucionalista, todo el entramado que envuelve a los procesos electorales contiene elementos substantivos que inciden en la dinámica política y en la más compleja senda hacia la consolidación democrática. Para evaluar esta situación relativamente reciente y crecientemente homogénea en América Latina es preciso analizar el significado de las elecciones desde una perspec-

(*) Universidad de Salamanca.

(1) En un reciente trabajo de opinión pública (Latinobarómetro,1995), a la pregunta de "¿Ud. cree, en términos generales, que las elecciones en su país son limpias o son fraudulentas?", los que consideraron limpias las elecciones fueron: Argentina: 68 por ciento; Brasil: 25 por ciento; Chile: 75 por ciento; México: 12 por ciento; Paraguay: 9 por ciento; Perú: 57 por ciento; Uruguay: 71 por ciento; y Venezuela: 18 por ciento. Por el contrario, preguntados sobre si "la manera como uno vota puede hacer que las cosas sean diferentes en el futuro" contestaron afirmativamente: $\mathrm{Ar}$ gentina: 69 por ciento; Brasil: 50 por ciento; Chile: 53 por ciento; México: 49 por ciento; Paraguay: 50 por ciento; Perú: 67 por ciento; Uruguay: 69 por ciento; y Venezuela: 47 por ciento. En cuanto a la pregunta sobre si "sin partidos no puede haber democracia" las respuestas afirmativas fueron: Argentina: 71 por ciento; Brasil: 47 por ciento; Chile: 57 por ciento; México: 50 por ciento; Paraguay: 47 por ciento; Perú: 53 por ciento; Uruguay: 77 por ciento; y Venezuela: 55 por ciento. tiva lo más interdisciplinar posible (2). En las páginas que siguen, se abordan aspectos relativos al necesario estudio de los sistemas electorales, contabilizando elementos como son la fórmula electoral y la relación teórica entre sistema electoral y gobernabilidad, también se analiza la relación existente entre leyes electorales y sistemas de partidos, para finalizar con la exposición de diferentes patrones derivados del comportamiento electoral tanto de los partidos latinoamericanos como del electorado en la década de 1990 sobre la base de fuentes empíricas.

La riqueza derivada del alto número de elecciones celebradas en condiciones de normalidad en América Latina ofrece una insólita posibilidad, en términos históricos, para llevar a cabo, con el soporte empírico derivado de dichos resultados, estudios innovadores sobre el sistema político en la línea de los realizados por Montero (1992) para España o por Shugart y Carey (1992) y Mainwaring y Shugart (1996) para la propia América Latina. Además de lo que significa este reto para el politólogo, se presentan diferentes expresiones que llevan, una vez más, a cuestionar la homogeneidad de la región, pero que también vienen a reforzar interpretaciones más o menos tradicionales sobre el universo partidista, sus peculiaridades y sus funciones distintas en los diversos países. Por último, igualmente se deducen "leyes" del comportamiento político que, en la mayoría de las situaciones, coinciden con las formuladas en otros ámbitos geográficos de mayor tradición de este tipo de estudios, y que, en las menos, llegan a plantear escenarios teóricos novedosos.

(2) Desde la perspectiva española, las aproximaciones más jurídicas y formales (Montero et ali, 1992; Fernández Segado, 1994) dejan paso a otras en las que la 6ptica se fija más en aspectos sistémicos. Este es el caso de recientes estudios sobre las elecciones salvadoreñas (Alcántara, 1994b), mexicanas (Martínez, 1995) y uruguayas (Crespo y Mieres, 1996), y los que se llevan a cabo en la presente publicación. 


\section{EL ESTUDIO DE LOS SISTEMAS ELECTORALES}

En América Latina, donde el juego electoral estuvo viciado por fraudes, proscripciones partidistas e incluso por su propio cuestionamiento como, se decía, "mecanismo formal carente de significado real y reflejo del vacío mundo de la política representativa", los estudios electorales apenas si tuvieron espacio en la reflexión académica. Este legado perdura en gran medida en la actualidad de suerte que la discusión en torno a los autores clásicos en la materia apenas si cala en foros reducidos separados de una realidad política cerrada y excluyente, cuando no marcadamente hostil. La oleada democratizadora de la década de 1980 puso en sintonía a la región, en lo atinente a las cuestiones electorales, con las corrientes neoinstitucionalistas que volvían la vista hacia los sistemas electorales como fuente interpretativa de sucesos en el seno del sistema político. Sin embargo, no será hasta la década de 1990 cuando la generalización de los procesos electorales libres y competitivos en todos los países latinoamericanos, salvo Cuba, permita la realización de análisis globales que dedujeran pautas de comportamiento diferenciadas.

\section{El cuestionado peso de la fórmula electoral}

Desde los trabajos seminales de Duverger (1951) y de Rae (1967) hasta los más recientes de Sartori (1994) y de Lijphart (1995), los análisis que abordan los sistemas electorales han avanzado notablemente. Con dificultad se han encontrado repercusiones de las lizas electorales en América Latina en dicha reflexión teórica. Uno de los criterios fundamentales usados tradicionalmente para clasificar los sistemas electorales, que es la fórmula electoral, esto es, el reparto de escaños según los votos, y que da origen a los conocidos sistemas mayoritario, de pluralidad y proporcional, apenas si ha tenido repercusión alguna en la historia de los sistemas políticos de los países latinoamericanos. Sin embargo, el hecho de que el propio carácter de estas fórmulas así como su insuficiencia analítica fuesen rebatidos a lo largo de la década de 1980 cuando en América Latina afloraba la democracia- y, además, fuese ganando aceptación la incorporación de sistemas mixtos, sirvió para que el debate llegase también a la región.

Rae (1967: 137-138) expresamente había sostenido que la principal diferencia entre las leyes (fórmulas) electorales yacía en el continuo sencillo entre proporcionalidad perfecta (donde no sucede la redistribución) y la desproporcionalidad extrema (donde los partidos fuertes se benefician más y los débiles sufren más). Este aspecto fue considerado irrelevante años más tarde por Rose (1984: 75) quien en un estudio con abundante trabajo empírico demostró que la diferencia total en el grado de proporcionalidad entre la fórmula de pluralidad y la de representación proporcional apenas si era de 8 puntos en la media de siete casos regidos por la primera y diecisiete por la segunda. Por el contrario, Nohlen (1984: 313) estimó que era errónea la visión de un continuo en los sistemas electorales por cuanto que los dos principios de representación existentes "deberían considerarse como antitéticos”. En esta discusión, América Latina, como campo de estudio, estaba ausente.

Una estricta fijación en la fórmula electoral no resolvía, por consiguiente, su peso real en el sistema electoral. Tanto los estudios citados de Rose como los de Nohlen, amparados en un sólido soporte empírico, conducían a posturas divergentes. El "resultado escéptico" que los datos de Rose alcanzaban no era, sin embargo, incompatible con la incuestionable afirmación de Nohlen del grado de oposición de ambas formulas. La solución a esta aparente paradoja debía incorporar otros aspectos sistémicos de la mecánica electoral. Tomando como base de estudio el panorama electoral en América Latina, donde se debe partir de la base de la adopción generalizada de la fórmula de representación proporcional, y considerando que al menos tres son los aspectos que deben considerarse, se añadía algo de luz al contencioso sobre la fórmula electoral. Los aspectos a tener en cuenta son:

a) El índice de electores/escaños que permite plantear una primera aproximación del hecho representativo nacional toda vez que cuanto más alto sea, mayores constricciones generará a la hora del reparto de los escaños. Se trata, en definitiva, de asegurar un "mínimo idóneo" por el que no sea enormemente difícil y costoso alcanzar un escaño. En América Latina (ver Cuadro I) se registran, en este sentido, dos características: en primer lugar, hay dos modelos bien marcados y un caso excepcional. Los primeros se refieren a aquellos casos cuyo índice es inferior a 50.000 electores/escaño y a los que se inserta en el segmento 50.000-100.000 electores /escaño. La excepción la constituye Brasil con un índice que se acerca a 200.000 electores/escaño. La segunda característica estriba en una tendencia a que este índice paulatinamente vaya creciendo en todos los casos, bien por el incremento del censo bien por la disminución del número de escaños que se viene registrando en las últimas reformas constitucionales (3).

\section{Cuadro I}

\section{Indices de electores/escaños}

\begin{tabular}{|c|c|c|c|c|}
\hline País & Año & Censo & $\begin{array}{l}\text { Número } \\
\text { Escaños }\end{array}$ & Indice \\
\hline Bolivia & 1989 & 2.136 .587 & 130 & 26.435 \\
\hline Costa Rica & 1990 & 1.692 .050 & 57 & 29.685 \\
\hline Honduras & 1989 & 2.366 .448 & 128 & 18.500 \\
\hline Guatemala & 1995 & 3.204 .955 & 116 & 27.630 \\
\hline Nicaragua & 1990 & 1.752 .088 & 92 & 19.000 \\
\hline Paraguay & 1989 & 2.226 .061 & 72 & 31.000 \\
\hline República Dominicana & 1990 & 3.275 .570 & 120 & 27.300 \\
\hline Uruguay & 1989 & 2.319 .022 & 99 & 23.425 \\
\hline Argentina & 1989 & 20.022 .072 & 254 & 78.830 \\
\hline Colombia & $\begin{array}{l}1990 \\
1994 \\
\end{array}$ & $\begin{array}{l}13.793 .566 \\
17.037 .771 \\
\end{array}$ & $\begin{array}{l}199 \\
163 \\
\end{array}$ & $\begin{array}{r}69.315 \\
104.500 \\
\end{array}$ \\
\hline Chile & 1989 & 7.556 .613 & 120 & 62.970 \\
\hline Ecuador & 1988 & 4.679 .684 & 71 & 65.911 \\
\hline México & $\begin{array}{l}1988 \\
1994 \\
\end{array}$ & $\begin{array}{l}38.074 .926 \\
45.729 .053 \\
\end{array}$ & $\begin{array}{l}500 \\
500 \\
\end{array}$ & $\begin{array}{l}76.150 \\
91.460 \\
\end{array}$ \\
\hline $\begin{array}{l}\text { Perú } \\
\text { Venezuela }\end{array}$ & $\begin{array}{l}1990 \\
1988 \\
1993 \\
\end{array}$ & $\begin{array}{l}9.923 .062 \\
9.185 .647 \\
9.975 .944 \\
\end{array}$ & $\begin{array}{l}180 \\
200 \\
201 \\
\end{array}$ & $\begin{array}{l}55.130 \\
45.928 \\
49.631 \\
\end{array}$ \\
\hline Brasil & $\begin{array}{l}1990 \\
1994 \\
\end{array}$ & $\begin{array}{r}83.817 .593 \\
94.782 .410 \\
\end{array}$ & $\begin{array}{l}503 \\
513 \\
\end{array}$ & $\begin{array}{l}166.700 \\
184.800 \\
\end{array}$ \\
\hline
\end{tabular}

Los cálculos de estos cuadros han sido realizados por Araceli Mateos sobre la base de datos electorales procedentes de: Boletín Electoral Latinoamericano. (1990-1995), Martínez (1995) y Nohlen (1993).

(3) Es el caso de las reformas habidas en Colombia, Chile, Guatemala y Perú que redujeron sensiblemente el número de diputados con respecto a la etapa constitucional anterior. 
El diferente esfuerzo nacional para alcanzar representación de las ofertas partidistas es, de esta manera, muy irregular. Si se lograra confirmar la hipótesis de que un menor índice acarrearía una cierta equiparación entre representación proporcional y mayoritaria, la fórmula electoral escogida en los países integrados en el primero de los modelos (aquel de índice inferior a 50.000) sería claramente irrelevante.

b) El número de circunscripciones electorales existente, su consiguiente tamaño en términos de puestos electivos y de electores y, de ahí, el nuevo índice de electores/escaños existente para cada una de ella. La vinculación de la circunscripción en la práctica totalidad de los países latinoamericanos a un espacio administrativo, como es la provincia, el estado o el departamento, cuya desigualdad poblacional es frecuente, introduce un sesgo inicial de notable desproporcionalidad. Esta situación apenas es corregida mediante el reparto de escaños por número habitantes toda vez que se suele fijar un mínimo de escaños por circunscripción con independencia de su tamaño.

c) La existencia de un "umbral electoral" por el que se quedaran fuera del reparto de escaños aquellas formaciones políticas que no alcanzaran un mínimo porcentual de sufragios es otra de las prácticas más extendidas en todos los casos latinoamericanos. Este umbral varía del 3\% al 5\%, penaliza a las fuerzas minoritarias, hace que un buen número de votos no alcancen cuotas de representación y, consiguientemente, introduce otro factor de desproporcionalidad. Este factor es aún si cabe más agudo en las grandes circunscripciones en las que el umbral marcado significa dejar fuera de la representación a decenas de miles de votantes.

\section{Desproporcionalidad}

Los diferentes sistemas electorales ejercen una gran influencia en los sistemas de partidos, de tal manera que, dependiendo de las características de la ley electoral de cada país se puede provocar, en un mayor o menor grado, una reducción del número efectivo de partidos electorales al convertirlos en parlamentarios. Es decir, dependiendo de las fórmulas utilizadas para la conversión de los votos obtenidos en unas elecciones en escaños, los países pueden presentar distintos grados de desproporcionalidad.

Por todo ello, y de acuerdo con Lijphart (1995: 103), la desproporcionalidad, en cuanto "desviación existente entre el porcentaje de escaños y el de votos de los partidos", es una consecuencia política de los sistemas electorales, que, "por medios mecánicos y psicológicos, reduce el número de partidos y aumenta la probabilidad de que puedan darse victorias de un partido mayoritario" (Lijphart, 1995: 123). Esta circunstancia se vincula con los propios efectos del presidencialismo. A pesar de que tiende a reducir el multipartidismo habida cuenta que la presidencia es el mayor premio político, notablemente, el índice de desproporcionalidad de los países latinoamericanos (ver Cuadro II) se sitúa en niveles inferiores a los de otros países con formas de gobierno parlamentarias (Lijphart, 1995: 187).
Cuadro II

Indices de desproporcionalidad en las elecciones legislativas

\begin{tabular}{lcc}
\hline País & Año & Indice de desproporcionalidad \\
\hline Bolivia & 1989 & 4,66 \\
\hline Colombia & 1990 & 0,47 \\
\hline Costa Rica & 1990 & 3,43 \\
\hline Chile & 1989 & 3,82 \\
& 1993 & 4,07 \\
\hline Ecuador & 1990 & 2,59 \\
\hline El Salvador & 1991 & 3,11 \\
& 1994 & 3,72 \\
\hline Guatemala & 1990 & 7,6 \\
& 1995 & 11,6 \\
\hline Honduras & 1989 & 2,34 \\
& 1993 & 2,84 \\
\hline México & 1991 & 2,61 \\
& 1994 & 7,66 \\
\hline Nicaragua & 1990 & 1,57 \\
\hline Paraguay & 1989 & 8,68 \\
\hline Perú & 1990 & 4,18 \\
& 1995 & 3,11 \\
\hline R. Dominicana & 1990 & 3,54 \\
\hline Uruguay & 1989 & 0,56 \\
\hline Venezuela & 1994 & 0,75 \\
\hline El indice aquí una & 3,37 \\
\hline
\end{tabular}

El índice aquí utilizado es de Bernard Grofman

$$
\sqrt{\frac{1}{n e p} \sum(V i-e i)^{2}}
$$

vi es el porcentaje de votos; ei es el porcentaje de escaños y nep es el número efectivo de partidos.

Los valores del índice de desproporcionalidad para los países de América Latina presentan una característica común para todos ellos consistente en que los efectos desproporcionales de sus sistemas electorales son menores en las elecciones celebradas en los primeros años analizados que en las siguientes, con la excepción de Perú que ve disminuir su índice de desproporcionalidad de 4,2 a 3,1.

Las mayores diferencias entre los índices de las distintas convocatorias electorales correponden a México con un índice de 1,8 en 1991 y 7,7 en 1994, y Guatemala con un índice de 7,6 en 1990 y de 11,6 en 1995. Los países donde la diferencia entre los índices de desproporcionalidad entre las diferentes elecciones son Uruguay, El Salvador y Chile. En cuanto a los países que, de manera constante, presentan una desproporcionalidad más pequeña son Uruguay y Colombia con índices inferiores a la unidad.

La paulatina incorporación de sistema mixtos, siguiendo el modelo del sistema electoral alemán (Morales y Alcántara, 1991), ha pretendido, entre otros objetivos, eliminar la desproporcionalidad. Este sistema ha ido adquiriendo un notable prestigio (Sartori, 1994: 75) a la hora de asegurar un equilibrio entre las ventajas de los sistemas de representación proporcional (el aprovechamiento máximo del voto al traducir la expresión popular en escaños) y de los sistemas de pluralidad (la personalización del candidato y su responsabilidad directa frente a su electorado). Así, a la inclusión inicial de este sistema en México en 1977 con el fin de limitar las victorias electorales mecánicas del PRI repartiendo los "escaños proporcionales" entre los partidos de oposición, siguió tres lustros más tarde la adopción del sistema en Venezuela envuelto en diferentes presiones para "estrechar los vínculos entre electores y elegidos, estimular los liderazgos locales y representar a las minorías" (Ramos, 1995: 155). Ahora es objeto de estudio en Uruguay. 
De este modo, complementariamente, se da una especial atención a la propia estructura del voto, tanto en lo que se refiere a su objeto (listas o individuos aislados) como al procedimiento (número de votos que tiene cada elector y tipo de voto -nominal, ordinal, numérico-); y a la estructura del distrito electoral con respecto a la naturaleza de su composición y a su magnitud. Todo ello, además, debe ser concebido en un marco que tenga en cuenta la situación histórica y socio-política específica bajo la que el sistema electoral debe operar y en conexión con la teoría democrática (Nohlen, 1984: 89).

\section{Sistema electoral y gobernabilidad}

El sistema electoral se ha señalado como una explicación complementaria responsable de problemas de desajustes en el sistema político que deriven en crisis de gobernabilidad. Su impacto más relevante en un sistema político se realiza sobre los partidos a quienes confiere representación efectiva en los distintos órganos pluripersonales de gobierno. Durante mucho tiempo se pensó en América Latina que los partidos tenían un carácter autónomo con respecto al sistema electoral. Si esto pudo ser así hasta la incorporación del sufragio universal, en la inestable segunda mitad del siglo XX el impacto de los sistemas electorales sobre los sistemas políticos tuvo un mayor reconocimiento (4).

Nohlen (1992) distingue dos efectos relevantes del sistema electoral sobre la gobernabilidad: el "efecto reductivo" obtenido por la comparación entre el número de partidos que compiten y el de partidos que obtienen mandatos parlamentarios; y el proceso de formación de mayorías (tanto en su vertiente estrictamente parlamentaria como en la presidencial -esto es del mismo "color" político que el Presidente-). Para el caso latinoamericano en concreto, Nohlen (1992:27) encuentra que los sistemas electorales ejercen un efecto moderado, "sea reductivo (sobre la cantidad de partidos) o sea desproporcionador (sobre la relación entre votos y escaños), de manera que su influencia en los sistemas de partidos no llega a determinar su estructura "en forma exclusiva y tajante".

Los sistemas electorales prefiguran, en buena medida, el sistema de partidos y, a su vez, pueden llegar a condicionar enormemente el rendimiento del sistema político, sobre todo en situaciones dominadas por una transición política. A pesar de haber recibido críticas en otras facetas, queda todavía en pié el sesgo persistente que, según Rae (1967: 134), tienen todos los sistemas electorales, y que elevó al rango de ley, de favorecer a los partidos fuertes en detrimento de sus competidores más débiles. Esta característica genera consecuencias diferentes en sistemas políticos con grados de consolidación democrática diversos y, a la vez, repercute en la propia credibilidad del sistema en cuanto a capacidad de poner verdaderamente en marcha algunas de las funciones tradicionales de los partidos. Además, èn la

(4) Es en Chile y Uruguay donde la diferente literatura reconoce un mayor impacto entre sistema electoral y sistema político. arena latinoamericana donde el presidencialismo es un condicionante notable, los partidos fuertes reciben un impulso extra del presidente o de los candidatos con claras posibilidades de serlo.

Ahora bien, la consolidación del universo partidista debía tener en cuenta, para el caso latinoamericano, entre otros factores (Alcántara, 1994), el punto de partida pretransicional en el que el nivel de "vigor partidista" cobraba importancia extrema. Por "vigor partidista" se entendía una calificación de la vida de los partidos políticos preexistente, medida por: la pluralidad de los mismos; la continuidad temporal de las formaciones partidistas; la atracción de una alta movilización electoral popular de apoyo; y la gestación de subculturas políticas.

Pero en un segundo escenario debía tener cabida, en primer término, el grado de movilización que logró conseguir la coalición social o política sobre la que se sostenía el régimen no democrático anterior $\mathrm{y}$, después, el tipo de institucionalización de éste. Combinando la movilización y la institucionalización de los regímenes no democráticos se obtiene una definición de casos que atienden bien a un nivel alto de ambas o a uno de perfil bajo.

La unión de los dos escenarios, a efectos de establecer el "clima" previo de los partidos políticos antes de la transición y la consecuente proyección en el periodo postransicional, permite establecer un plano con los dos ejes conformados por el "vigor partidista" y por la combinación recién enunciada entre movilización e institucionalización que dan paso a los cuatro tipos siguientes: el cuadrante dominado por valores máximos de ambas variables se denomina de "facilidad poliárquica" puesto que da cabida a sociedades que recogen tradiciones de partidismo democrático con alta capacidad movilizadora y una fuerte tendencia hacia la institucionalización expresamente formalizada. En este espacio, el nuevo régimen democrático se ve conformado por sistemas de partidos fuertes, ampliamente presente en la sociedad, con capacidad intermediadora y catalizadores fundamentales de la vida política. El cuadrante dominado por los valores mínimos se define de "imposibilidad poliárquica", siendo la tónica general la de la debilidad extrema, cuando no inexistencia, del sistema de partidos. Los cuadrantes que conforman la diagonal transversal generan una situación dominada por la combinación de un alto "vigor partidista" con una reducida institucionalización y movilización, este es el espacio intitulado "proclividad poliárquica";la política, sesgada por el déficit histórico institucional, adquiere caracteres personalistas a los que contrabalancea el sistema de partidos que desempeña un papel bastante activo en el nuevo régimen democrático. La forma opuesta o de "dificultad poliárquica", contempla la escasa capacidad de un tradicionalmente débil sistema de partidos para neutralizar los liderazgos caudillistas que alcanzan ciertas cotas de institucionalización y de apoyo social.

En un paso subsiguiente, camino de la consolidación del sistema político democrático, el grado de institucionalización de los partidos es también objeto de preocupación. De forma que recae en cuatro elementos: el modelo de competición entre los partidos y la diferencia que les separa en número de escaños entre una elección y otra; el nivel de in- 
cardinación de los partidos en la sociedad medido por su pervivencia a lo largo del tiempo y la fidelidad de los electores; la percepción, en términos de legitimidad, que tienen los ciudadanos de los partidos y de las elecciones; y la estructura y el funcionamiento interno de los partidos (Mainwaring y Scully, 1995).

Tanto en un caso como en el otro, junto con aspectos ligados a la estructura social, al surgimiento de clivajes o a la legitimidad de los procedimientos de la democracia representativa, aparece el factor institucional como determinante del sistema de partidos. En este sentido, Sartori (1994: 27) ha denunciado lo equivocado de la asunción de que los sistemas electorales no fueran variables independientes y de que sus efectos fueran inciertos. Las leyes electorales son las variables que se tienen en cuenta a continuación.

\section{LA INFLUENCIA DE LAS LEYES ELECTORALES EN LOS SISTEMAS DE PARTIDOS}

\section{Introducción}

La influencia de las leyes electorales en los sistemas de partidos se ejerce, básicamente, a lo largo de tres dimensiones que son: el número de partidos; la capacidad de sobreprimar a uno o varios partidos en detrimento de otros; y la fortaleza de los propios partidos. Las variables que entran en liza en las leyes electorales y que tienen que ver con estas tres dimensiones son la votación en sí misma considerada, la circunscripción y la fórmula electoral (Rae, 1967: 16 y ss.). A menudo, el sistema electoral elegido combina las tres dimensiones y en otras ocasiones sólo dos de ellas, pero esta circunstancia no es óbice para que ahora puedan examinarse por separado, habida cuenta de los impactos especiales que pudieran tener sobre el sistema político. Como premisa relevante debe señalarse que el éxito real de un partido político es obtener "parcelas de representación", ésto es escaños que puedan garantizarle una presencia en el cuerpo legislativo mayor. Según esta afirmación, quedan de lado aquellos comicios en los que, por la naturaleza de la elección, hay un único vencedor y que se sitúan en el ámbito de un determinado tipo de poderes ejecutivos.

\section{El número de partidos}

El número de partidos se encuentra condicionado, en primer lugar, por el sistema de representación utilizado. La ley formulada por Duverger (1951) de que el sistema de pluralidad favorece el bipartidismo mientras que el sistema proporcional desarrolla el pluripartidismo tiene todavía bastante vigencia aunque parece cuestionada por la realidad según se incorporan nuevos casos de estudio y si bien, por otra parte, Sartori (1994) se ha ocupado de su desarrollo. Se puede afirmar que siempre que se aplica el sistema de representación de pluralidad hay bipartidismo, pero no siempre un sistema de representación proporcional genera multipartidismo. En segundo lugar, como ya ha quedado dicho, el número de partidos queda influído por la aplicación de un umbral mínimo en los casos de representación proporcional, de manera que los partidos que no lo superan quedan excluídos del reparto de escaños parlamentarios, a pesar de que les correspondiesen según un criterio de estricta proporcionalidad. La experiencia latinoamericana es bastante interesante en este apartado por cuanto que se ha puesto de relieve cómo puede darse una situación de bipartidismo "a pesar del" sistema de representación proporcional, como ha sucedido tradicionalmente en Argentina, Colombia, Costa Rica, Honduras y Venezuela, entre otros países.

La relación del número de partidos con el tipo de sistema político es imprecisa e inconsistente. Mientras que se ha llegado a afirmar que un menor número de partidos favorece un tipo de sistema mayoritario, según la terminología de Lijphart, más proclive al juego simple gobierno-oposición, paralelamente se ha constatado que la representación proporcional se inclina hacia un tipo de sistema consociacional en el que la negociacón es casi permanente. En el caso latinoamericano, donde el presidencialismo introduce la noción del "oficialismo", convive la lógica gobierno-oposición -típica del sistema mayoritario, generada desde el poder ejecutivo, con las necesidades que a veces surgen en el poder legislativo de cooperar entre grupos diversos para alcanzar mayorías suficientes -aspecto éste clásico del consociacionalismo-.

\section{No todos los partidos son iguales, algunos "son más iguales" que otros}

En cuanto a la capacidad de sobreprimar a unos partidos frente a otros, es obvia en el caso de las fórmulas de pluralidad y mayoritaria. No obstante, en el sistema de representación proporcional puede igualmente darse si no se respeta la regla de la estricta proporcionalidad (igual porcentaje de voto popular que de escaños adquiridos), y se produce como consecuencia de la aplicación de tres mecanismos: la formula matemática del reparto de los "restos", que en muchos casos puede beneficiar a los partidos con mayor número de votos (como sucede cuando se aplica el método D'Hondt). La modificación artificial de los límites de las circunscripciones para agrupar electorados "convenientes" (gerrymandering). Y la proporción del número de electores por escaño a distribuir, que en muchas circunstancias tiende a ser distinta según las circunscripciones. En este sentido, Nohlen (1984:84) resalta que este mecanismo comporta la misma incidencia en dañar la proporcionalidad pura que el establecimiento de umbrales mínimos señalado anteriormente. Esta situación ya había sido denunciada por Rae (1967: 138) al indicar que, como regla general, la combinación de la fórmula de la representación proporcional con las circunscripciones grandes producen con mayor proximidad resultados proporcionales, mientras que el sistema de pluralidad combinado con circunscripciones pequeñas produce la mayor desproporcionalidad.

Con respecto al tipo de impacto de la gestación artificial de partidos sobreprimados en relación con sus efectos sobre el sistema político es también contradictorio. Los beneficios 
de la existencia de mayorías sustanciales que supongan en un principio cierta estabilidad para el sistema político pueden transformarse a largo plazo en tumores con efectos negativos sobre el mismo. En una determinada coyuntura de crisis el sistema político puede verse bloqueado hasta la extenuación por dichas mayorías ajenas a la realidad social. Los casos del Partido Revolucionario Institucional mexicano,del Partido Colorado paraguayo y de los Partidos Liberal y Conservador colombianos son claramente determinantes en este aspecto.

\section{La fortaleza de los partidos políticos}

La posibilidad de fortalecer a los partidos de acuerdo con los criterios electorales escogidos es fruto de la utilización de instrumentos que restringen enormemente la capacidad de decisión del elector. Entre ellos, el más conocido y usado son las listas cerradas y bloqueadas elaboradas en los "estados mayores" de los partidos y frente a las que el elector no tiene capacidad alguna de ejercer ni opciones de prelación, ni de combinación de sus candidatos preferidos entre listas de partidos distintos. Este instrumento genera otra situación muy criticada, que es la de alejar a los electores de sus representantes, por una parte, y la de atrapar al político en la red partidista dado que todo se lo debe a ella.

También en este apartado la relación del grado de fortaleza, así adquirida, con el carácter que tome el sistema político es ambigua. Mientras que las listas cerradas y bloqueadas es un mecanismo básico para dotar de solidez y consistencia al nuevo sistema de partidos en una situación transicional, su flexibilización mediante la introducción de opciones de preferencia es una práctica que puede luego llegar a ser detestable por su significado a la hora de impulsar políticas clientelísticas dentro de los partidos. Además, la imposición de diputados cuneros ajenos a la realidad de la circunscripción y con una elevada capacidad de escaparse del control de sus electores contrasta con la necesidad de romper con prácticas caciquiles que pudieran perdurar en el caso de confiar demasiado en la clase política local. El reconocimiento de estos problemas en América Latina ésta en el origen de los intentos de reforma electoral que se llevan a cabo, siendo el más efectivo el producido en 1993 en Venezuela ya descrito anteriormente.

\section{COMPORTAMIENTO ELECTORAL EN LA DECADA DE 1990 EN AMERICA LATINA}

De las diferentes aproximaciones que se pueden llevar a cabo a la hora de analizar el comportamiento electoral en América Latina, a la luz de los puntos anteriormente considerados, a continuación se abordan únicamente los relativos al número de partidos, a la concentración del voto, a la fragmentación partidista y a la volatilidad registrada. Para ello, el escenario institucional donde se lleva a cabo este análisis es el conformado por las elecciones al poder legislativo $\mathrm{y}$, en aquellos casos en que hubiere bicameralismo, más concretamente a la Cámara de Diputados. Esta opción es limitada en muchos países latinoamericanos por el excesivo "tirón" electoral de las elecciones presidenciales, pero, a efectos del estudio de la representación efectiva de los partidos, su fuerza y su tendencia a la institucionalización, es la más adecuada. En cualquier caso, se trata de una primera aproximación que pretende animar el estudio del comportamiento electoral y de sus implicaciones sobre el sistema político desde una perspectiva novedosa a la luz del análisis comparado.

\section{Número efectivo de partidos}

La utilización del número efectivo de partidos según la conocida fórmula (5) es oportuna para establecer cómo se traduce el tamaño de los respectivos partidos en liza en un dígito concreto que permita llevar a cabo una perspectiva comparada, todo ello a pesar de las duras críticas vertidas a este instrumento (Sartori, 1994). Este análisis (Cuadro III) posibilita plantear que la evolución sufrida en América Latina en las dos últimas elecciones al Congreso realizadas en los diferentes países muestra una tendencia clara a la estabilización o, en su defecto, a la disminución de dicho número. La disminución del número efectivo de partidos no indica más que la posibilidad de hablar de una cierta estabilidad en el sistema de partidos (6) o de un realineamiento partidista. Unicamente el caso de Venezuela destaca por su manifiesto incremento, traduciendo la inestabilidad vivida en dicho país como consecuencia de la crisis de los partidos tradicionales y del surgimiento de la opción electoral que apoyó a Rafael Caldera en su carrera presidencial. En Paraguay, donde también se registra un incremento en el número de partidos, este es debido a la superación en las elecciones de 1993 de la anómala situación generada por el comportamiento hegemónico del Partido Colorado en los comicios de 1989; en 1993 la liza electoral se movió en el marco de una mayor competencia real.

En todos los casos, el número efectivo de partidos en términos de porcentaje de votos (número efectivo de partidos electorales) es siempre ligeramente superior al medido sobre porcentajes de escaños (número efectivo de partidos parlamentarios), lo que se traduce en una evidencia de la capacidad reductora de los partidos por parte de las diferentes fórmulas electorales. Diferencia que se manifiesta claramente en Chile, Ecuador y Guatemala, donde es aproximadamente de dos puntos. Estos países, además, coinciden con aquellos en los que el número es mayor, reflejando a la vez una competitividad superior entre los mismos y una mayor igualdad relativa entre los resultados obtenidos. Sin embargo, esta tendencia no acontece en el caso de Perú y de

(5) La fórmula del número efectivo de partidos de Laakso y Taagapera es: $N=1 / \Sigma v i^{2}$ ó $N=1 / \Sigma e i^{2}$ donde $v$ es el porcentaje de votos del partido $i$, y $e$ es el porcentaje de escaños del partido $i$.

(6) Se ha señalado oportunamente que, con la excepción de Chile entre 1932 y 1973, todos los regímenes democráticos latinoamericanos que han durado al menos un cuarto de siglo han tenido un número efectivo de partidos inferior a 3. Tal ha sido el caso de Costa Rica, Colombia, Venezuela y Uruguay (Mainwaring y Scully, 1995: 33). 
Paraguay en las elecciones de 1990 y de 1989 respectivamente, ya que el número efectivo de partidos parlamentarios es mayor al electoral debido a la existencia de coaliciones electorales cuyos integrantes toman posesión de sus escaños posteriormente en representación de sus propios partidos, provocando que a la hora de decidir entre unas candidaturas haya un número menor de partidos pero después se aumente al traducirse en diputados.

Los casos de Colombia, Costa Rica, El Salvador, Honduras y Uruguay muestran unas diferencias pequeñas entre los números de partidos electorales y congresuales de suerte que su fórmula electoral no contribuye a reducir el número de partidos manteniendo una mayor proporcionalidad.

Los países latinoamericanos pueden encuadrarse en tres tipos de categorías. La primera integra a aquellos casos en los que el número efectivo de partidos es inferior a 2,40 , aproximándose a la lógica de los sistemas bipartidistas, y que recoge a Colombia, Costa Rica, Honduras, México y Paraguay (los índices inferiores a 2 de este país en 1989 se debieron a la presencia hegemónica del Partido Colorado en el Congreso). La segunda da cabida a los sistemas de partidos de número situado entre 2,40 y 4,00, o de pluralismo limitado, en la que se incluyen El Salvador, República Dominicana, Uruguay y Bolivia. En la última categoría, con un número efectivo de partidos mayor a cuatro se encuentran Guatemala, Ecuador, Chile y Brasil, correspondiendo a la categoría de Sartori de pluralismo extremo.

En términos de media, el número de partidos en los países de América Latina es ligeramente superior al dado para el periodo 1977-89 para países europeos (Montero, 1992: 278). España, en dicho período contaba con 3,87 partidos electorales y con 2,66 partidos parlamentarios, situándose en una posición baja de una tabla en que los extremos venían dados por el Reino Unido (2,97 y 2,12 respectivamente) y por Bélgica $(7,27$ y 6,42 respectivamente)

\section{Concentración del voto}

El análisis de la concentración del voto permite visualizar la traducción de los flujos electorales en escaños, medidos porcentualmente, de los dos partidos más votados y, en un segundo nivel, de los cuatro partidos más votados. De esta manera, se complementa el contenido del apartado anterior. Al partir de la existencia de una competencia partidista mínima de dos contendientes (los números efectivos de partidos de las últimas elecciones son siempre superiores a dos), esta forma de observación facilita constatar el diferente grado de concentración del voto entre los actores principales.

De los quince países estudiados (ver Gráfico 1), cuatro presentan una alta concentración del voto en los dos partidos principales. En estos países: Colombia, Costa Rica, Nicaragua (7) y Paraguay, como promedio,, nueve de cada diez electores optaron por uno de los dos principales partidos. Además, en otros cuatro países: El Salvador, Guatema-

(7) Si bien UNO, la formación vencedora en las elecciones, era una coalición electoral y no un partido político. la, México, y Uruguay, el electorado concentró su voto en el mismo porcentaje en los cuatro partidos principales.

\section{Gráfico 1}

Concentración del voto en los dos y cuatro principales partidos con inayor número de escaños*

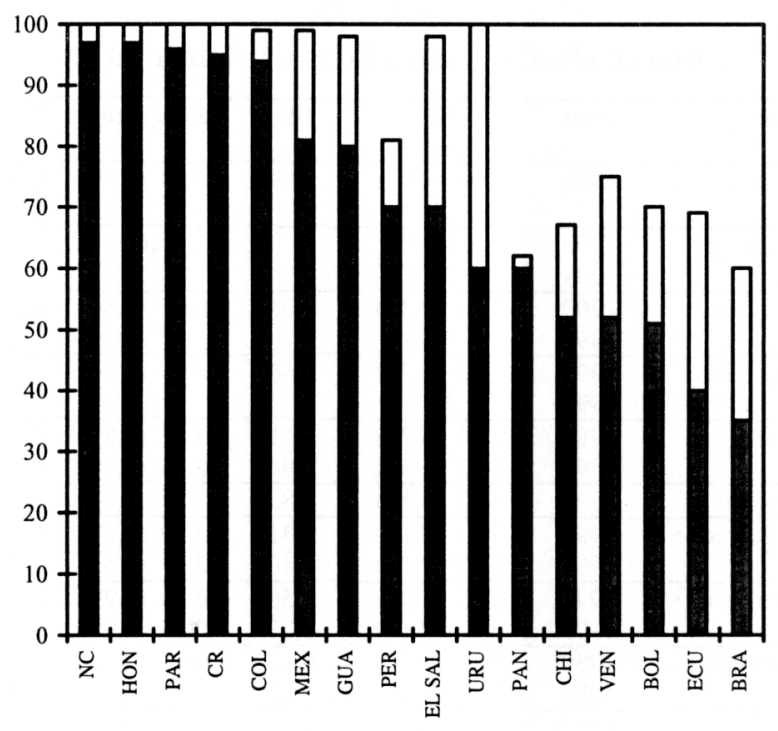

* Los porcentajes pertenecen a las últimas elecciones legislativas celebradas en cada uno de los países.

Los países que presentan una menor concentración del voto en los dos partidos principales son Brasil, Ecuador, Venezuela y Chile, aunque esta concentración aumenta considerablemente al tenerse en cuenta los cuatro partidos principales. Cabe destacar el caso de Panamá, puesto que sin presentar una concentración de voto uy alta en los dos partidos mayoritarios, no aumenta más que un $2 \%$ de los escaños en los cuatro partidos mayoritarios.

\section{Fragmentación partidista}

El índice de fragmentación (8) evalúa, con mayor claridad, cual es la relación existente entre el número de partidos y su tamaño así como la competitividad interpartidista. Este índice se refiere "a la probabilidad de que dos votantes seleccionados al azar opten por diferentes partidos en una determinada elección, o a la de que dos parlamentarios de una misma legislatura, también seleccionados al azar, pertenezcan a partidos distintos" (Montero, 1992: 272). El valor mínimo, cero, delata la existencia de una única opción partidista y, por consiguiente, la ausencia de competencia. El valor máximo, uno, indica que hay tantas opciones partidistas como casos. El valor intermedio $(0,5)$ traduce la existencia de dos partidos.

En primer lugar hay que señalar que, al igual que en el número efectivo de partidos, pueden establecerse diferencias

(8) La fórmula del índice de fragmentación es: $F=1-\Sigma V i^{2}$ ó $F=1-\Sigma e i^{2}$, donde $V i$ es el porcentaje de votos del partido $i$, y ei es el porcentaje de escaños del partido i. 
entre la fragmentación electoral y la parlamentaria. Para el caso de los países latinoamericanos (Cuadro III), la fragmentación electoral es también ligeramente mayor a la parlamentaria;a excepción, igualmente, de los casos de Paraguay y de Perú que en las elecciones de 1989 y de 1990, respectivamente, la fragmentación parlamentaria fue mayor que la electoral.

\section{Cuadro III}

Número efectivo de partidos y fragmentación

\begin{tabular}{|c|c|c|c|c|c|}
\hline País & Año & $\begin{array}{c}\mathrm{N}^{\circ} \\
\text { de partidos }\end{array}$ & Dif. & Fragment. & Dif. \\
\hline Bolivia & 1989 & $\begin{array}{c}5,00(3,91) \\
(4,69)\end{array}$ & 1,09 & $\begin{array}{c}0,80(0,74) \\
(0,78)\end{array}$ & 0,06 \\
\hline Brasil & $\begin{array}{l}1990 \\
1994\end{array}$ & $\begin{array}{l}(8,70) \\
(8,27)\end{array}$ & & $\begin{array}{l}(0,88) \\
(0,87)\end{array}$ & \\
\hline Colombia & $\begin{array}{l}1990 \\
1994\end{array}$ & $\begin{array}{c}2,23(2,19) \\
(2,20)\end{array}$ & 0,04 & $\begin{array}{c}0,55(0,54) \\
(0,54)\end{array}$ & 0,01 \\
\hline Costa Rica & $\begin{array}{l}1990 \\
1994\end{array}$ & $\begin{array}{c}2,57(2,21) \\
(2,30)\end{array}$ & 0,36 & $\begin{array}{c}0,61(0,54) \\
(0,56)\end{array}$ & 0,07 \\
\hline Chile & $\begin{array}{l}1989 \\
1993\end{array}$ & $\begin{array}{ll}7,85 & (5,34) \\
6,91 & (4,97)\end{array}$ & $\begin{array}{l}2,51 \\
1,94\end{array}$ & $\begin{array}{ll}0,87 & (0,81) \\
0,85 & (0,79)\end{array}$ & $\begin{array}{l}0,06 \\
0,06\end{array}$ \\
\hline Ecuador & 1990 & $8,40(6,58)$ & 1,82 & $0,88(0,84)$ & 0,04 \\
\hline El Salvador & $\begin{array}{l}1991 \\
1994\end{array}$ & $\begin{array}{ll}3,33 & (3,02) \\
3,52 & (3,06)\end{array}$ & $\begin{array}{l}0,31 \\
0,46\end{array}$ & $\begin{array}{ll}0,70 & (0,67) \\
0,72 & (0,67)\end{array}$ & $\begin{array}{l}0,03 \\
0,05\end{array}$ \\
\hline Guatemala & $\begin{array}{l}1990 \\
1995\end{array}$ & $\begin{array}{ll}6,93 & (4,43) \\
4,87 & (2,60)\end{array}$ & $\begin{array}{l}2,50 \\
2,27\end{array}$ & $\begin{array}{ll}0,85 & (0,77) \\
0,79 & (0,61)\end{array}$ & $\begin{array}{l}0,08 \\
0,18\end{array}$ \\
\hline Honduras & $\begin{array}{l}1989 \\
1993\end{array}$ & $\begin{array}{ll}2,12 & (2,00) \\
2,33 & (2,10)\end{array}$ & $\begin{array}{l}0,12 \\
0,23\end{array}$ & $\begin{array}{ll}0,52 & (0,50) \\
0,57 & (0,52)\end{array}$ & $\begin{array}{l}0,02 \\
0,05\end{array}$ \\
\hline México & $\begin{array}{l}1991 \\
1994\end{array}$ & $\begin{array}{ll}2,39 & (2,21) \\
3,08 & (2,30)\end{array}$ & $\begin{array}{l}0,18 \\
0,78\end{array}$ & $\begin{array}{ll}0,58 & (0,55) \\
0,67 & (0,56)\end{array}$ & $\begin{array}{l}0,03 \\
0,11\end{array}$ \\
\hline Nicaragua & 1990 & $2,18(2,05)$ & 0,13 & $0,54(0,51)$ & 0,03 \\
\hline Panamá & 1994 & $(4,30)$ & & $(0,76)$ & \\
\hline Paraguay & $\begin{array}{l}1989 \\
1993\end{array}$ & $\begin{array}{c}1,69(1,88) \\
(2,57)\end{array}$ & $-0,19$ & $\begin{array}{c}0,41(0,47) \\
(0,61)\end{array}$ & $-0,06$ \\
\hline Perú & $\begin{array}{l}1990 \\
1995 \\
\end{array}$ & $\begin{array}{ll}4,77 & (5,77) \\
3,30 & (2,78)\end{array}$ & $\begin{array}{c}-1,00 \\
0,52 \\
\end{array}$ & $\begin{array}{ll}0,79 & (0,82) \\
0,69 & (0,64)\end{array}$ & $\begin{array}{l}-0,03 \\
0,05\end{array}$ \\
\hline $\begin{array}{l}\text { Repúblic. } \\
\text { Dominica. }\end{array}$ & $\begin{array}{l}1990 \\
1994 \\
\end{array}$ & $\begin{array}{ll}3,75 & (3,05) \\
2,69 & (2,43) \\
\end{array}$ & $\begin{array}{l}0,70 \\
0,26\end{array}$ & $\begin{array}{ll}0,73 & (0,67) \\
0,62 & (0,59) \\
\end{array}$ & $\begin{array}{l}0,06 \\
0,03\end{array}$ \\
\hline$\overline{\text { Uruguay }}$ & $\begin{array}{l}1989 \\
1994\end{array}$ & $\begin{array}{ll}3,37 & (3,35) \\
3,43 & (3,30)\end{array}$ & $\begin{array}{l}0,02 \\
0,13\end{array}$ & $\begin{array}{ll}0,70 & (0,70) \\
0,71 & (0,69)\end{array}$ & $\begin{array}{c}0 \\
0,02\end{array}$ \\
\hline Venezuela & $\begin{array}{l}1988 \\
1993\end{array}$ & $\begin{array}{c}3,36(2,82) \\
(4,53)\end{array}$ & 0,54 & $\begin{array}{c}0,70(0,64) \\
(0,77)\end{array}$ & 0,06 \\
\hline
\end{tabular}

Entre paréntesis figuran los indices relativos al ámbito de la Cámara de Diputados de suerte que solamente recogen a los partidos con representación parlamentaria. Dif. alude a la diferencia electoral y congresual.

Dentro de las situaciones en las que es posible comparar los resultados de dos elecciones, se observa que la diferencia entre fragmentación electoral y parlamentaria en el último año en el que tuvieron lugar las elecciones tiende a ser mayor que la de las elecciones anteriores. Sin embargo, Chile y Uruguay son los dos países donde la diferencia entre ambas fragmentaciones se mantiene constante, lo que parece indicar que mantienen una considerable estabilidad en las preferencias de su electorado no produciéndose cambios importantes electorales relacionados con fenómenos de realineamiento o concentración de preferencias y desalineamiento en sus sistemas políticos.Este hecho avala la interpretación tradicional del alto grado de institucionalización de su sistema de partidos.

La fragmentación parlamentaria se ha incrementado notablemente en Colombia y Venezuela, aunque no llega a los valores de Brasil y, en segundo término, de Ecuador, lo que traduce la crisis de sus sistemas de partidos. Por el contrario, ha disminuido, también de forma acusada, en Guatemala y Perú, países que encontraron serias dificultades al comienzo de la década de 1990 en estabilizar su sistema de partidos.
Los países latinoamericanos se mueven en un amplio abanico que, al igual que en el apartado anterior, no permite poder referirse a la existencia de patrones comunes regionales. Brasil, Ecuador y Chile contabilizan los valores más altos de fragmentación partidista, superior a 0,80 , frente a Honduras, Costa Rica, Colombia y México que aportan los más bajos, inferiores a 0,63 . En términos comparativos con los casos europeos, los índices de fragmentación latinoamericanos son ligeramente superiores, aunque bien es cierto que el periodo estudiado para Europa es mayor (1977-89). En este sentido, obsérvese que para dicho periodo los índice de fragmentación electoral y parlamentaria españoles fueron del 0,74 y del 0,63 respectivamente (Montero, 1992: 273). Ahora bien, la fragmentación partidista es más problemática en las democracias presidenciales que en las parlamentarias toda vez que aquellas carecen de los mecanismos institucionales para alcanzar a formar coaliciones (Mainwaring y Scully, 1995: 33).

\section{La volatilidad}

Los índices de volatilidad (9) muestran "los cambios electorales agregados que se producen en un sistema de partidos entre dos elecciones sucesivas y que se deben a transferencias individuales del voto" (Montero, 1992: 283). La volatilidad cristaliza, por tanto, la orientación del voto y la competitividad interpartidista gracias a los cambios habidos en los porcentajes de voto entre dos elecciones. De una forma agregada como la aquí estudiada pone de relieve la estabilidad de las preferencias electorales hacia un sistema de partidos determinado. Una volatilidad alta representa que el electorado ha desplazado substantivamente sus preferencias de unos partidos a otros lo que viene derivado normalmente de que se hubieran producido un importante número de altas y/o bajas dentro de los partidos contendientes. Por otra parte, cuanto más bajos son los índices de volatilidad puede hablarse de una mayor estabilización y consolidación del sistema de partidos en esos países.

A pesar de no disponer de todos los índices de volatilidad correspondientes a aquellos países de América Latina en los que han tenido lugar elecciones legislativas sucesivas, pueden establecerse tres categorías nacionales a la luz de los índices obtenidos (ver Cuadro IV). La primera categoría incluiría a Venezuela y a México con volatilidades altas superiores al valor 18; en estos casos el alto índice alcanzado no significa una falta de continuidad partidista o ausencia de partidos políticos fuertes, más bien es la respuesta del electorado ante la situación de crisis que se vive. La segunda categoría daría cabida a Uruguay, Guatemala, Honduras y a Perú, con índices intermedios comprendido entre 11,2 y 8,5 . Finalmente se encontraría el caso de Chile con una volatilidad de 6,6 lo que traduce un alto grado de consolidación del sistema de partidos y de estabilidad de las preferencias electorales.

(9) La fórmula del índice de volatilidad agregada es $V T=\Sigma[P i t-P i(t+1)] / z$. Pi representa el apoyo electoral, en porcentajes sobre el voto válido para el partido $i$ en los tiempos $t$ y $t+1$, es decir, en dos elecciones seguidas. 
Cuadro IV

Indices de volatilidad

\begin{tabular}{lcc}
\hline País & Periodo & Indice de volatilidad \\
\hline Chile & $1989-1993$ & 6,6 \\
El Salvador & $1991-1994$ & 12,6 \\
Guatemala & $1990-1995$ & 10,7 \\
Honduras & $1989-1993$ & 9,6 \\
México & $1991-1994$ & 18,6 \\
Perú & $1990-1995$ & 8,5 \\
Uruguay & $1989-1994$ & 11,2 \\
Venezuela & $1988-1993$ & 21,0 \\
\hline
\end{tabular}

Con las limitaciones derivadas de que el presente estudio abarca un periodo limitado en el que todavía no se pueden dar cabida "ciclos electorales", los valores de volatilidad de los países latinoamericanos están en consonancia con los de otros casos europeos (Montero, 1992: 288). La volatilidad mexicana para el periodo 1991-94 es prácticamente igual a la española para el periodo 1977-1989 (que es la más alta de entre 17 casos europeos considerados).

\section{CONCLUSIONES}

Los comicios celebrados en la década de 1990 en América Latina han supuesto, de manera generalizada, la implantación de prácticas poliárquicas continuadas a escenarios caracterizados históricamente por la inexistencia de las mismas, sus irregularidades o sus interrupciiones cíclicas. Esta nueva situación permite la introducción de elementos de análisis propios de la politología electoral habituales en el estudio de las democracias consolidadas. Los índices clásicos que miden el impacto de las elecciones en el sistema de partidos, en cuanto a su número ponderado, concentración del voto y fragmentación o en la volatilidad de las preferencias del electorado, pueden comenzar a calcularse aportando, a pesar de la inmadurez de muchas de las prácticas poliárquicas, de la pobreza e imprecisión de los resultados electorales ofrecidos y del reducido número de casos analizable, aproximaciones primeras. Los resultados que ofrecen dichos índices que, de alguna manera, traducen la expresión del comportamiento electoral de los ciudadanos, han puesto de relieve la existencia de una gran heterogeneidad de los mismos. Lo que lleva a la constatación de la vigencia de diferentes modelos y al sinsentido de referencias regionales de carácter globalizante.

Por otra parte, el predominio de la institución presidencial, en detrimento del poder legislativo, y la hiperconcentración de la atención pública en las elecciones presidenciales frente a otro tipo de comicios, es un factor que hace que, aparentemente, sean marginales estudios como el que aquí se quiere esbozar. A pesar del sesgo introducido en la política por el presidencialismo y del influjo masivo de los medios de comunicación (especialmente los televisivos) tanto en la forja de liderazgos como en las campañas electorales, el universo partidista sigue desempeñando un papel relevante. Los procesos de formación y selección de personal político, la articulación y representación de intereses, la gestación de canales para la participación política y la propia operatividad procedimental del sistema político en el seno de numerosas instituciones co- legiadas, son funciones necesarias de los partidos. De ahí que su revalorización, através de las elecciones, siga teniendo un significado. Además, el impacto de un sistema de partidos fuerte, traducido básicamente por índices reducidos de volatilidad y alta concentración del voto, en la consolidación del sistema político democrático es incuestionable. De esta suerte, los resultados aquí ofrecidos para los casos de Uruguay, Chile, Costa Rica y Honduras en la década de 1990 avalan un correcto camino hacia la consolidación democrática. Circunstancia que es más cuestionable para los restantes casos estudiados.

El número efectivo de partidos presente en la vida política latinoamericana traduce realidades plurales de las sociedades. Estas visiones contrastan con las más monolíticas que, superficialmente, muestran el predominio de factores en clave de "antipolítica", mediante los que se explican liderazgos coyunturales antipartido. Incluso dicho número choca con la tendencia que, a priori, podría conducir el presidencialismo favorable a una lógica dual gobierno-oposición, y por ende al bipartidismo. La generalización de la fórmula de representación proporcional ha ayudado, inequívocamente, a definir este estado de cosas. Una visión similar se obtiene al observar los niveles de fragmentación existentes. A través de ambos índices, en cualquier caso, no se infiere, ni se contradice, la bondad del universo existente.

Es en este terreno, típico latinoamericano, de relacionar presidencialismo con representación proporcional donde se puede obtener, con el tiempo, importantes resultados teóricos. Por el momento, el universo estudiado muestra resultados tan heterogéneos que no permiten establecer correlaciones fuertes ni del número de partidos, ni de la fragmentación partidista, ni de la volatilidad electoral con ambas variables. Sin embargo, su presencia institucional, cuando se compara América Latina con Europa, aparece como el elemento diferenciador.

\section{BIBLIOGRAFIA CITADA}

ALCÁNTARA, MANUEl (1994). "Análisis comparado del papel de los partidos en los procesos de transición política". En Silvia Dutrénit y Leonardo Valdés (comp.) El fin de siglo y los partidos políticos en América Latina. Instituto Mora. Itzapalapa. México. 19-34.

- (1994b). "Las 'elecciones del siglo' salvadoreñas". Revista de Estudios Políticos. Madrid. 85. 323-337.

Boletín EleCtORAL LATINOAMERICANO. 1993-95. CAPEL-IIDH. San José de Costa Rica.

CRESPO, ISMAel y PABlo Mieres (1996). "Las elecciones uruguayas de 1994: continuidad en la trasnformación del sistema partidista". Revista de Estudios Políticos. Madrid.

DUVERGer, MAURICE (1951). Les partis politiques. A. Colin. Paris. Edición en español: (1965) Los partidos politicos. Fondo de Cultura Económica. México.

FERNÁNDEZ SEGADO, FRANCISCO (1994). "La representatividad de los sistemas electorales". Cuadernos de CAPEL. San José de Costa Rica. 37

LATINOBARÓMETRO (1995). Apoyo Opinión y Mercado S.A. Lima 
LIJPHART, AREND (1995). Sistemas electorales y sistemas de partidos. Centro de Estudios Constitucionales. Madrid.

MAINWARING, SCOTT (1995). "Presidencialismo, multipartidismo y democracia: la difícil combinación”. Revista de Estudios Políticos. Madrid. 88 115-144.

- y Timothy Scully (1995). Building Democratic Institutions. Party System in Latin America. Stanford University Press. Stanford.

- y Matthew S. Shugart (1996). Presidentialism and Democracy in Latin America. Cambridge University Press. Cambridge.

MARTíneZ, ANTONIA (1995). "Las elecciones federales mexicanas de agosto de 1994". Revista de Estudios Políticos. Madrid. 88 311-339.

MONTERO, JosÉ RAMÓN (1992). "Las elecciones legislativas”. En Ramón Cotarelo (comp.). Transición política y consolidación democrática. España (19751986). Centro de Investigaciones Sociológicas. Madrid. 243-297.

Montero, José Ramón; Manuel Alcántara; Miguel REVEnga; IsMAel CRESPO; ANTONiA MARTínez Y PABLO OÑATE (1992). "La legislación electoral de Iberoamérica: una aproximación comparada". En Procesos electorales en Iberoamérica: Organización, administración y ejecución. Ministerio del Interior-Ministerio de Asuntos Exteriores. Madrid. 285-507.
Morales, Pilar y Alcántara, ManUel (1991). "La ley electoral en el proceso de unificación alemana". Revista de Estudios Políticos. Madrid. 72 279-300.

NOHLEN, DiETER (1984). “Two Incompatible Principles of Representation”. En Lijphart y Grofman (1984). 83-89.

- (1992). Sistemas electorales y gobernabilidad. Working Paper 63. Institut de Ciéncies Polítiques i Socials. Barcelona.

- (Coord.)(1993). Enciclopedia electoral Latinoamericana y del Caribe. Instituto Interamericano de Derechos $\mathrm{Hu}-$ manos. San José de Costa Rica.

RaE, Douglas (1967). The Political Consequences of Electoral Laws. Yale University Press. New Haven.

RAMOS, MARÍA LuISA (1995). De las protestas a las propuestas. Identidad, acción y relevancia política del movimiento vecinal en Venezuela. Nueva Sociedad. Caracas.

ROSE, RICHARD (1984). "Electoral Systems: A Question of Degree or of Principle?”. En Lijphart y Grofman (1984). 73-81.

SARTORI, GIOVANNI (1994). Comparative Constitutional Engineering. An Inquiry into Structures, Incentives and Outcomes. New York University Press. New York.

Shugart, Matthew S. Y John M. Carey (1992). Presidents and Assemblies: Constitutional Design and Electoral Dynamics. Cambridge University Press. Cambridge.

\section{RESUMEN}

En este artículo el autor aprovecha la reciente experiencia electoral latinoamericana para realizar un estudio de los sistemas electorales de la región, analizando aspectos como la fórmula electoral y la relación entre el sistema electoral y la gobernabilidad. Seguidamente estudia la relación entre leyes electorales y sistemas de partidos, así como las tendencias del comportamiento del electorado y de los partidos latinoamericanos. Finalmente se extraen conclusiones globales a la luz de los recientes procesos electorales.

\section{ABSTRACT}

In this article, the author takes the most recent electoral experience in Latin America to make a study on the different electoral systems of the region, by analyzing various aspects like the electoral formula and the connection between electoral system and governability. Next, the article studies the connection between electoral laws and party system, as well as the Latin America elector' \& parties' behaviourial trends. Finally, several global conclusions are inferred in the light of the most recent electoral processes. 